\title{
Le plafonnement des indemnités de licenciement sans cause réelle et sérieuse sur la sellette
}

\section{Marion Galy}

\section{(2) OpenEdition}

1 Journals

\section{Édition électronique}

URL : https://journals.openedition.org/rdctss/1687

DOI : $10.4000 /$ rdctss. 1687

ISSN : 2262-9815

Éditeur

Centre de droit comparé du travail et de la sécurité sociale

\section{Édition imprimée}

Date de publication : 1 avril 2019

Pagination : 210-213

ISSN : 2117-4350

\section{Référence électronique}

Marion Galy, « Le plafonnement des indemnités de licenciement sans cause réelle et sérieuse sur la sellette ", Revue de droit comparé du travail et de la sécurité sociale [En ligne], 1 | 2019, mis en ligne le 01 novembre 2021, consulté le 13 novembre 2021. URL : http://journals.openedition.org/rdctss/1687 ; DOI : https://doi.org/10.4000/rdctss. 1687

\section{cc)}

Revue de droit comparé du travail et de la sécurité sociale est mise à disposition selon les termes de la Licence Creative Commons Attribution - Pas d'Utilisation Commerciale - Pas de Modification 4.0 International. 


\section{LE PLAFONNEMENT DES INDEMNITÉS DE LICENCIEMENT SANS CAUSE RÉELLE ET SÉRIEUSE SUR LA SELLETTE}

Le plafonnement des indemnités pour défaut de cause réelle et sérieuse est-il en train de vivre ses derniers instants? Mise en place par l'Ordonnance relative à la prévisibilité et à la sécurisation des relations de travail en septembre 2017, ${ }^{1}$ cette mesure a été relativement mal accueillie par les organisations syndicales et par une large part de la doctrine travailliste. Elle consiste à limiter le pouvoir d'appréciation du juge dans la fixation de l'indemnité de licenciement sans cause réelle et sérieuse en instaurant un plafond dont le montant varie selon l'ancienneté du salarié. Ce plafonnement illustre en réalité, une fois de plus, la défiance qui s'opère depuis quelques années à l'égard du juge prud'homal ${ }^{2}$ et s'ajoute à des mesures telles que l'instauration de référentiels guidant l'indemnisation du juge au stade de la conciliation ${ }^{3}$ ou le raccourcissement des délais de prescription. ${ }^{4}$ Ces différentes réformes ont, sinon pour objet, au moins pour effet, d'éviter aux employeurs d'avoir à se présenter devant le juge (prescription ou découragement des salariés au regard de l'encadrement des indemnités) et d'atténuer ses pouvoirs lorsque la confrontation s'avère inévitable. ${ }^{5}$

De nombreuses stratégies judiciaires ont alors été mises en œuvre pour contrer ce nouveau dispositif et, après quelques tentatives peu concluantes, les plaideurs ont réussi à trouver dans les textes internationaux, et plus particulièrement dans les articles 10 de la Convention $n^{\circ} 158$ de l'OIT et 24 de la Charte sociale européenne révisée, des arguments juridiques de poids. Plusieurs conseils de prud'hommes ont en effet récemment écarté dans différents litiges l'article L.1235-3 du Code du travail - qui consacre le plafonnement - au motif de sa contrariété aux textes précités. Si ces jugements n'ont évidemment pas vocation à «faire jurisprudence », leur étude n'en présente pas moins d'intérêt. Du caractère plus ou moins convaincant des arguments avancés (I) dépend en effet la remise en cause pérenne du plafonnement des indemnités de licenciement sans cause réelle et sérieuse (II).

\section{I - UN PLAFONNEMENT ÉCARTÉ EN PREMIÈRE INSTANCE}

Les premières remises en cause. Pas moins de trois conseils de prud'hommes ont successivement refusé d'appliquer l'article L.1235-3 du Code du travail. À l'étude, la motivation des jugements s'avère plus ou moins convaincante. Dans le premier d'entre

1 Art. L.1235-3 du Code du travail.

2 Sur cette défiance, voir M. Grévy et P. Henriot, "Le juge, ce gêneur... », Revue Droit Travail 2013, p. 173. F. Guiomard, «La justice du travail confrontée aux politiques de flexicurité », Revue Droit Travail 2013, p. 52.

3 D. Boulmier, «Faciliter la conciliation prud'homale... mais pour qui ?», Droit social 2013, p. 837, sp. p. 839.

4 Th. Pasquier, "Où mènent les mauvais chemins...? », in Controverse «Faut-il caler le temps de la justice sur celui de l'entreprise ?», Revue Droit Travail 2014, p. 153.

5 P. Henriot, « Le juge privé de sentences?», Droit ouvrier 2018, p. 160. 
eux, rendu le 13 décembre 2018 par le conseil de prud'hommes de Troyes, ${ }^{6}$ l'argument défendu est le suivant: le plafonnement serait inconventionnel car contraire à l'article 10 de la Convention $n^{\circ} 158$ de l'Organisation internationale du travail - qui garantit au juge la possibilité d'ordonner le versement d'une "indemnité adéquate ou toute autre forme de réparation considérée comme appropriée " lorsque la réintégration du salarié n'est pas possible en cas de licenciement injustifié - et à l'article 24 de la Charte sociale européenne, qui consacre le "droit des travailleurs licenciés sans motif valable à une indemnité adéquate ou à une autre réparation appropriée ". Pour mettre à l'écart le plafonnement des indemnités pour licenciement sans cause réelle et sérieuse, le conseil de prud'hommes d'Amiens s'est uniquement fondé sur l'article 10 de la Convention $n^{\circ} 158$ de l'OIT et a affirmé que l'indemnité versée ne pouvait " être considérée comme étant appropriée et réparatrice du licenciement sans cause réelle et sérieuse ». ${ }^{7}$ Les plus récents jugements, rendus par le conseil de prud'hommes de Lyon les 21 décembre 2018 et 7 janvier 2019 sont plus ambivalents. ${ }^{8}$ La motivation du premier se révèle très expéditive ; les conseillers prud'hommes se sont certes référés à l'article 24 de la Charte sociale européenne pour fixer le montant de l'indemnité, mais aucune allusion n'est faite à une éventuelle inconventionnalité de l'article L.1235-3 du Code du travail pour justifier sa mise à l'écart. Le jugement du 7 janvier 2019 est à ce titre plus convaincant et se rapproche dans sa motivation de celle retenue par les juges troyens; y est affirmé la contrariété du plafonnement aux articles 10 de la Convention n 158 de l'OIT et 24 de la Charte sociale européenne révisée.

Vers une remise en cause généralisée du plafonnement? Répondre à cette question s'avère particulièrement délicat lorsqu'on sait que peu de temps auparavant, en septembre 2018, le conseil de prud'hommes du Mans a refusé d'appliquer l'article 24 de la Charte sociale européenne et a considéré l'article L.1235-3 du Code du travail conforme à l'article 10 de la Convention n 158 de l'OIT'. S'il s'avère en conséquence impossible de prédire dans quel sens se prononceront les autres conseils de prud'hommes, on peut toutefois, sans trop s'avancer, imaginer que les conseils de salariés vont s'engouffrer dans cette brèche et multiplier les demandes en ce sens. Il faut dire que les autres voies de mise en échec du plafond sont assez réduites. L'article L.1235-3-1 du Code du travail permet certes au juge de s'affranchir des plafonds, mais les conditions posées rendent cette voie assez étroite. Le licenciement doit en effet être entaché de l'une des nullités listées dans l'article : violation d'une liberté fondamentale, faits de harcèlement moral ou sexuel ou licenciement discriminatoire notamment. Par ailleurs, le succès d'une question prioritaire de constitutionnalité semble compromis puisque la disposition légale précitée a déjà été soumise et validée par le Conseil constitutionnel à deux reprises : lors de l'adoption de la loi d'habilitation à légiférer par voie d'ordonnance, ${ }^{10}$ puis lors de la loi de ratification. ${ }^{11}$ Sous bénéfice de ces observations, on mesure dès lors tout l'intérêt de l'argument conventionnel en ce qu'il demeure ouvert et qu'il permet d'écarter le barème en toutes circonstances. Jusqu'à ce que la Cour de cassation se prononce sur la pertinence de cet argument, une recrudescence du contentieux, que ce dispositif avait justement pour objet de limiter, est donc à prévoir. ${ }^{12}$

6 CPH Troyes, 13 déc. 2018, RG F 18/00036 : Lexbase Hebdo, éd. S 2019, n 767, obs. S. Tournaux.

7 CPH Amiens, 19 déc. 2018, RG F 18/00040.

8 CPH Lyon, 21 déc. 2018, RG F 18/01238 - CPH Lyon, 7 janv. 2019, RG n 15/01398.

9 CPH Mans, 26 sept. 2018, RG F 17/00538.

10 Cons. const., 7 sept. 2017, $n^{\circ}$ 2017-751 DC.

11 Cons. const., 21 mars 2018, n 2018-761 DC : Dr. soc. 2018, p. 718, obs. D. Baugard et J. Morin.

12 En ce sens : C. Geiger, « Note sous CPH Troyes, 13 déc. 2018 », Droit ouvrier 2019, p. 52. 


\section{II - VERS UNE REMISE EN CAUSE DÉFINITIVE DU PLAFONNEMENT}

L'attente d'un positionnement de la Cour de cassation. Comme le rappelle la plupart des commentateurs, ${ }^{13}$ la pérennité des solutions adoptées par ces conseils de prud'hommes dépendra de la position qu'adoptera in fine la Chambre sociale de la Cour de cassation. La jurisprudence ne peut en effet émaner de n'importe quelle décision de justice, rendue dans n'importe quelles conditions. La solution invoquée doit bénéficier " du support d'une décision dotée d'une particulière autorité ". ${ }^{14}$ Or, "les particularités de l'organisation juridictionnelle française conduisent à privilégier de ce point de vue, les arrêts du Conseil d'État, pour le droit public et ceux de la Cour de cassation, pour le droit privé, ces juridictions ayant le pouvoir d'imposer aux autres juges de leur ordre une interprétation déterminée de la règle de droit ${ }^{15}$. II s'écoulera ainsi plusieurs mois avant que les affaires précédemment exposées ne soient soumises (si elles le sont) au contrôle de la Chambre sociale de la Cour de cassation. Rendus en premier ressort, ces jugements devront au préalable faire l'objet d'un appel. La Cour de cassation pourrait toutefois être amenée à se prononcer sur la conventionnalité du plafonnement des indemnités pour licenciement sans cause réelle et sérieuse plus rapidement. La Haute juridiction peut en effet connaître de moyens d'inconventionnalité, pour la première fois, à la condition qu'ils constituent des moyens de pur droit ou qu'ils soient nés de la décision attaquée. ${ }^{16}$ Or, à notre sens, la question de la conformité de l'article L.1235-3 du Code du travail aux articles 10 de la Convention n 158 de l'OIT et 24 de la Charte sociale européenne révisée suppose seulement d'opérer un contrôle de compatibilité entre deux normes et ne nécessite en conséquence pas que la Cour de cassation se prononce sur des éléments factuels. Afin de réduire encore davantage la période d'incertitude dans laquelle se trouvent actuellement salariés et employeurs, les juges du fond pourraient par ailleurs solliciter l'avis de la Chambre sociale de la Cour de cassation. Les juges du fond ont en effet la possibilité de saisir l'une des chambres de la Cour de cassation afin de prévenir la survenance de divergences de jurisprudences. ${ }^{17}$ Pour recourir à un tel mécanisme, il est seulement exigé que le juge soit amené à trancher une question de droit nouvelle se posant dans de nombreux litiges. ${ }^{18}$ L'avis a pour but d'éviter que des juridictions du fond ne " se prononcent dans des sens divers, sinon opposés $"{ }^{19}$ Au regard de cette condition, le recours à cette procédure d'avis, relativement à la conventionnalité du plafonnement, semble en conséquence des plus justifié.

Une confirmation très probable de l'inconventionnalité par la Cour de cassation. Comme précédemment indiqué, la date à laquelle la Cour de cassation se positionnera sur cette inconventionnalité demeure incertaine. Cet état de fait n'empêche pour autant pas de mesurer les chances (ou risques, selon de quel point de vue on se place !) de confirmation d'une telle inconventionnalité par la Chambre sociale. Sur ce point, le raisonnement s'opérera en deux temps. Ce n'est qu'après avoir admis l'applicabilité directe des textes internationaux en cause que la Chambre sociale de la Cour de cassation pourra

13 Not. S. Tournaux, op. cit.

14 J.-L. Aubert et É. Savaux, Introduction au droit et thèmes fondamentaux du droit civil, $17^{\mathrm{e}}$ éd., coll. Université, Sirey 2018, p. 188.

15 Ibid.

16 Rapport annuel de la Cour de cassation 2013.

17 Art. L.441-1 à L.441-3 du Code de I'organisation judiciaire. Art. 1031-1 à 1031-7 du Code de procédure civile.

18 R. Libchaber, "La saisine pour avis, une procédure singulière dans le paysage jurisprudentiel ", Revue Trimestrielle Droit civil 2003, p. 157.

19 G. Canivet, «La Cour de cassation et les divergences de jurisprudence », in P. Ancel et M.-C. Rivier (dir.), Les divergences de jurisprudence, Publ. Univ. Saint Étienne 2003, coll. Droit, p. 141, sp. p. 155. 


\section{FRANCE}

se prononcer sur la contrariété de l'article L.1235-3 - et du plafonnement qu'il consacre aux dispositions internationales précitées.

Aucun argument dirimant ne semble s'opposer au succès de la première étape. Comme l'a très justement rappelé le Professeur Sébastien Tournaux, il faut ici distinguer l'applicabilité directe des dispositions de droit international de leur effet direct. ${ }^{20}$ En l'espèce, seule l'applicabilité directe de l'article 10 de la Convention n 158 de l'OIT et de l'article 24 de la Charte sociale européenne révisée est en cause, et non leur effet direct, puisqu'il ne s'agit pas d'appliquer une méthode d'indemnisation établie par ces articles mais de confronter ces derniers à l'article L.1235-3 du Code du travail. Or, dans plusieurs arrêts déjà, la Cour de cassation a eu l'occasion d'admettre l'applicabilité directe des articles de la Convention n ${ }^{\circ} 158$ de I'OIT. ${ }^{21}$ Par ailleurs, si la Cour de cassation n'a, à notre connaissance, jamais reconnu l'applicabilité directe de l'article 24 de la Charte sociale européenne, le Conseil d'État l'a toutefois admis en $2014,{ }^{22}$ et il serait surprenant que la Cour de cassation prenne le contrepied de cette décision dans un contexte marqué par le dialogue entre les deux ordres. ${ }^{23}$

Le succès de la seconde étape semble plus incertain. II ressort d'une lecture combinée des articles 10 de la Convention $n^{\circ} 158$ et de l'article ${ }^{24}$ de la Charte sociale européenne que si le plafonnement des indemnités n'est pas en lui-même condamnable, le législateur doit cependant mettre en mesure les juges de délivrer une indemnisation adéquate ou appropriée. Sur ce point, la Cour pourrait parfaitement retenir l'argumentation défendue par les juges manceaux et considérer qu'entre les planchers et plafonds ainsi fixés, le juge français bénéficie d'une marge d'appréciation, au-delà du seul critère de l'ancienneté. Une telle argumentation semble toutefois peu tenable lorsqu'on observe le montant des plafonds correspondant aux anciennetés les plus faibles. Si un salarié, présentant une ancienneté supérieure à trente années, peut prétendre à une indemnité dont le montant est compris entre trois et vingt mois de salaires, il en va différemment des salariés disposant d'une ancienneté inférieure à une année. Pour ces derniers, le plafond est fixé à un mois de salaire. Dans une telle hypothèse, il semble difficile d'affirmer que le plafonnement permet véritablement aux juges d'apprécier les situations individuelles des salariés injustement licenciés afin de réparer de manière «adéquate » le préjudice qu'ils ont subi. C'est d'ailleurs ce qu'ont parfaitement mis en évidence les conseillers prud'homaux lyonnais dans leur jugement. Selon eux, « une ancienneté faible n'exclut pas la nécessité d'indemniser le salarié en fonction notamment; d'une situation personnelle à la suite d'une perte d'emploi (âge, situation de famille, handicap de suspension...) ; et/ou d'une situation professionnelle rendant la recherche d'un nouvel emploi plus difficile (éloignement géographique, spécialités rares...) ; et/ou d'un préjudice professionnel réel, plus lourd que l'ancienneté ». ${ }^{24}$ II ne reste plus qu'à attendre que la Chambre sociale confirme cette contrariété aux textes internationaux que nous pressentons.

20 Sur cette distinction : J.-F. Akandji-Kombé, « De l'invocabilité des sources européennes et internationales du droit social devant le juge interne », Droit social 2012, p. 1014.

21 Cass. soc., 14 févr. 2018, n 16-20.987 - Cass. soc., 11 janv. 2012, n 10-17.945 - Cass. soc., $1^{\text {er juillet }}$ 2008, n07-44.124.

$22 \mathrm{CE}, 7^{\circ}$ et $2^{\circ}$ s-s-r., 10 févr. 2014, $n^{\circ} 358992$, publié au recueil Lebon.

23 A. Morin-Galvin, La convergence des jurisprudences de la Cour de cassation et du Conseil d'État : contribution au dialogue des juges en droit du travail, col. Bibliothèque Droit social, t. 60, LGDJ 2013, $818 \mathrm{p}$.

24 CPH Lyon, 7 janv. 2019, RG n 15/01398. 